\title{
Numerical Analysis Receiving/Transmitting Mechanisms of ZnO/Ag Nanoantennas
}

A. García-Barrientos ${ }^{1}$, F. R. Castillo-Soria ${ }^{1}$, M. A. Cardenas-Juarez ${ }^{1}$, V. I. Rodríguez-Abdala ${ }^{2}$, F. J. Gonzalez ${ }^{3}$ and J. E. Sánchez ${ }^{3}$

1. Faculty of Science, Universidad Autónoma de San Luis Potosí, SLP, Mexico.

2. Department of Electronics and Communications, Universidad Autónoma de Zacatecas, Mexico

3. LANCYTT, Universidad Autónoma de San Luis Potosí, SLP, Mexico

The fabrication, characterization and numerical analysis receiving/transmitting mechanisms of $\mathrm{ZnO} / \mathrm{Ag}$ nanoantenas are very important nowadays, because it is in the group II-VI metal oxide semiconductor with a wide direct band gap [1]. Semiconductor nanostructures are desirable for the modern electronics, nanophotonics, quantum circuitry, plasmonics and energy conversion applications as well as for fundamental science. Specially, in nanophotonics and plasmonics, optical nanoantennas can reduce the breach between photons and semiconductor emitters or detectors in nanoscale. Electrical and optical characteristics of $\mathrm{ZnO}$ films are pretty similar like $\mathrm{GaN}, \mathrm{ZnS}$, and compound semiconductors, and also because this material has different applications such as solid-state light sources and detectors in the blue and UV spectral range [2-3]. For applications in electronics and optics field, $\mathrm{ZnO}$ has interesting properties such as magnetic, piezoelectric, and semiconductor. It has a high electrical conductivity and a high optical gain at ambient temperature. In consequence by these properties, thin films $\mathrm{ZnO}$ has found numerous potential applications in fields such as, light emitting diodes, most gas sensors, and solar cells. For example, in the ref. [4] was reported the crystalline orientation analysis at the $\mathrm{ZnO} / \mathrm{Ag}$ interface, where $\mathrm{ZnO}$ rods grown on lateral faces of the pentagonal cross sectional area of silver nanowires were assembled in a hierarchical nanoantenna. This kind of nanoantenna is a promising alternative of plasmonic rf-nanoantennas for engineering light emission because of their low-loss nature in the spectrum [5-7].

In this paper, the numerical analysis receiving/transmitting mechanisms of $\mathrm{ZnO} / \mathrm{Ag}$ nanoantenas was carried out. In this case, a Yagi-Uda, with 11 elements, type nanoantenna was used to simulate the radiation patterns from 1-5 GHz. In the figure 1, we can see the SEM images of the ZnO thin films (a) and $\mathrm{ZnO} / \mathrm{Ag}$ hierarchical nanoantennas (b), reported in the ref. [4], where the pentagon geometry can be seen. In the figure 2, we can see the optimized antenna pattern at the design frequency at $5 \mathrm{GHz}(\mathrm{a})$, and to obtain a better insight into the behavior in the two orthogonal planes, we also plotted the normalized magnitude of the electric field in the E and H-planes, i.e. azimuth $=0$ (b) and 90 (c) deg, respectively. The results show that the nanoantennas might be capable of transmitting and/or receiving electromagnetic waves when stimulated with a modulated RF signal, especially at high frequency signals.

[1] Jongmin Kim, et al., Appl. Phys. Lett., vol. 102, no. 18, 183901, 2013.

[2] M.A. Contreras, Thin Solid Films, vol. 204, 403-404 pp., 2002.

[3] T. Ben Nasr, et al., Thin Solid Films, vol. 500, 4-8 pp., 2006.

[4] J. E. Sanchez, et al., Journal of Applied Physic, vol. 117, 034306 , 2015

[5] Sorias O, et al., Nano Lett,. vol. 17(10):6011-6017, 2017

[6] Peter M, et al., Nano Lett,. vol. 17(7):4178-4183, 2017

[7] Feng T., et al., J Phys Condens Matter., 2018

The authors acknowledge funding from the CONACYT-Mexico, research projects grant numbers 169062 and 204419. F. J. González would like to acknowledge support from Project 32 of "Centro 
Mexicano de Innovación en Energía Solar" and by the National Laboratory Program from CONACYT through the Terahertz Science and Technology National Lab (LANCYTT).

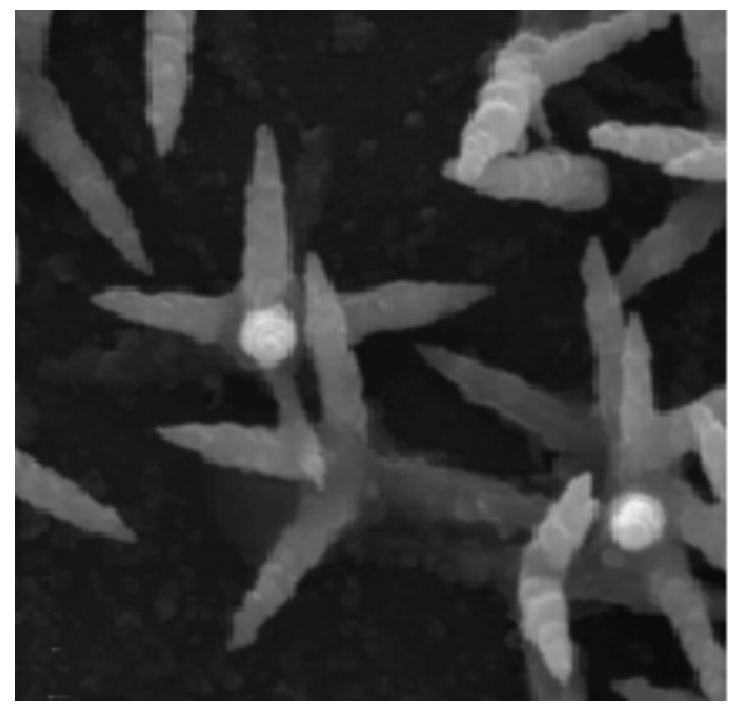

(a)

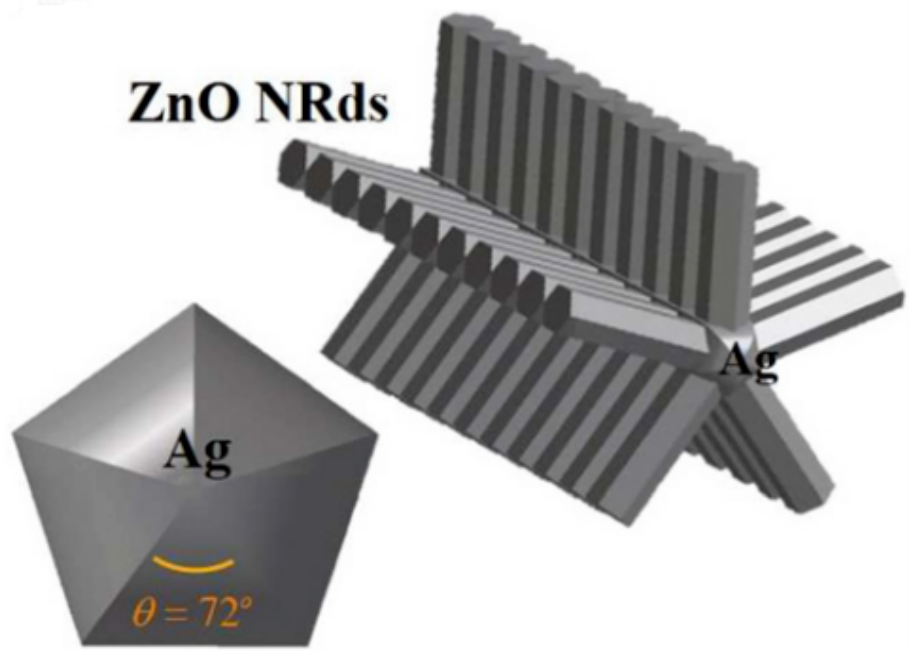

(b)

Figure 1. SEM photos of the samples surfaces of $\mathrm{ZnO}$ thin films (a) and $\mathrm{ZnO} / \mathrm{Ag}$ hierarchical nanoantennas (b).

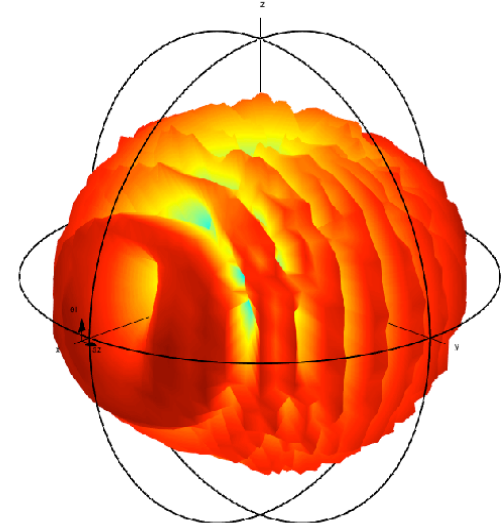

(a)

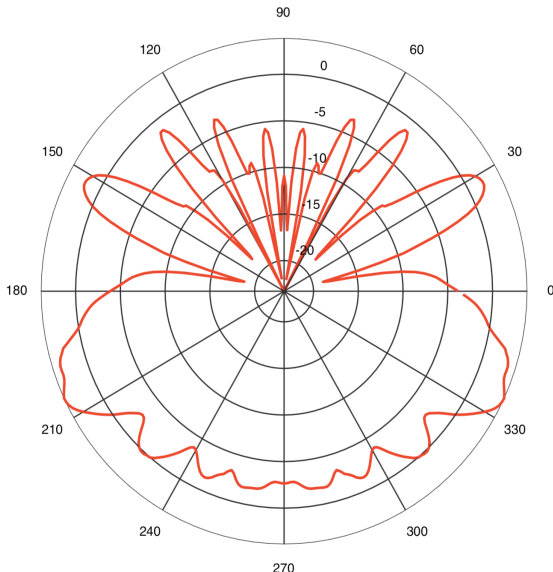

(b)

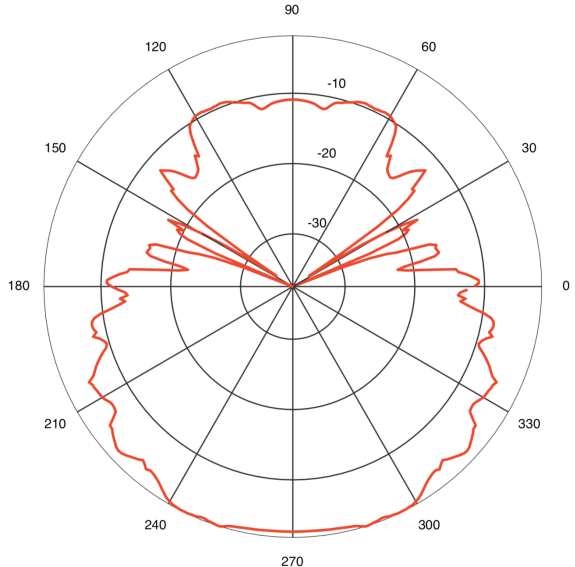

(c)

Figure 2. Yagi-Uda antenna 3D radiation pattern at the frequency $5 \mathrm{GHz}$ (a). The normalized magnitude of the electric field in the $\mathrm{E}$ and H-planes, azimuth $=0$ (b) and 90 (c) deg. 